\title{
A Avaliação do Rendimento Acadêmico de Estudantes em Modalidade de Ensino Remoto Durante a Pandemia de COVID-19 em Manaus
}

\section{Evaluation of Academic Performance of Students in Remote Learning during Covid-19 Pandemics in Manaus}

\author{
Xadreque Vitorino Macuácua*a; Angela Maria Araújo De Lima; ${ }^{\text {a }}$ Karem Teles Freitasa ; Lauro dos Santos Santarém \\ Junior $^{\text {a }}$ S Sídia Maria Ambrósio de Oliveira ${ }^{\text {a }}$ Henrique dos Santos Pereira ${ }^{a}$
}

aUniversidade Federal do Amazonas, Programa de Pós-Graduação em Ciências do Ambiente e Sustentabilidade na Amazônia. AM, Brasil.

*E-mail: xadreque14@gmail.com

\begin{abstract}
Resumo
Em decorrência da crise sanitária global provocada pela Pandemia de COVID-19, os sistemas dos vários níveis do ensino tiveram que se adaptar rapidamente e de modo precário, substituindo-se a modalidade presencial pela remota, por meio de ferramentas digitais. O presente estudo tem como objetivo analisar a relação entre a qualidade da ambiência vivenciada pelos estudantes em condições de isolamento doméstico e seu rendimento acadêmico no ensino remoto, aferidas por autoavaliação. Tomaram-se como sujeitos da pesquisa estudantes de graduação e Pós-graduação, em uma amostra de conveniência de 62 indivíduos maiores de 18 anos, de ambos os gêneros, na cidade de Manaus, Amazonas. Para coleta dos dados foi utilizado um formulário eletrônico compartilhado por aplicativo de mensagem. Os dados foram analisados por método gráfico e de regressão linear simples e múltipla. As componentes da avaliação da ambiência com piores desempenhos foram o compartilhamento do ambiente de estudo e dos equipamentos, o nível de ruído, a interrupção do sinal de internet e o trabalho simultâneo. O rendimento escolar foi considerado satisfatório na avaliação dos estudantes entrevistados, exceto no quesito interatividade. Resultados indicam que a qualidade ambiência interfere positivamente no aproveitamento, porém essa relação é influenciada pelo nível do curso e pelo gênero, vez que essa relação foi significativa somente para o nível de graduação e para estudantes do gênero masculino. Os desafios provocados pela transição educacional abrupta têm implicações negativas no desempenho e motivação dos estudantes.
\end{abstract}

Palavras-chave: Ensino. Ambiência. Aproveitamento dos Estudantes.

\begin{abstract}
As a result of the global health crisis caused by the Covid-19 pandemic, the systems of the various levels of education had to adapt quickly and in a precarious way, replacing the face-to-face modality with the remote one, through digital tools. This study aims to analyze the relationship between the quality of the environment experienced by students in conditions of domestic isolation and their academic performance in remote education, measured by self-assessment. Undergraduate and graduate students were taken as research subjects, in a convenience sample of 62 individuals over 18 years of age, of both genders, in the city of Manaus, Amazonas. For data collection, an electronic form shared by a message application was used. Data were analyzed by graphical method and simple and multiple linear regression. The ambience evaluation components with the worst performances were the sharing of the study environment and equipment, the noise level, the internet signal interruption and the simultaneous work. School performance was considered satisfactory in the evaluation of the interviewed students, except for the item interactivity. Results indicate that ambience quality positively affects achievement, but this relationship is influenced by the level of the course and by gender, since this relationship was significant only for the undergraduate level and for male students. The challenges brought about by the abrupt educational transition have negative implications for the students' performance and motivation.
\end{abstract}

Keywords: Teaching. Ambience. Students'Performance.

\section{Introdução}

Em 31 de dezembro de 2019, o Mundo tomou conhecimento do registro de um surto de pneumonia virial de causa desconhecida, na cidade de Wuhan, na China. O novo vírus foi denominado SARS-CoV-2 e em tempos de globalização e mundialização, foi rapidamente propagado pelo Mundo, provocando uma Pandemia global (CAVALCANTE, 2020). Em 30 de janeiro de 2020, o DiretorGeral da Organização Mundial da Saúde - OMS declarou o novo surto de corona vírus uma emergência de saúde pública de interesse internacional, o mais alto nível de alarme da OMS. A Pandemia desencadeou uma crise de saúde pública capaz de alterar estruturas socioeconômicas locais, regionais e globais (DIAS; PINTO, 2020; LIMA; BUSS; PAES-SOUSA, 2020). Nesse tempo, milhões de pessoas foram obrigadas a permanecer em isolamento social, provocando um período de profundas reflexões em torno dos profundos impactos provocados pelas mudanças socioeconômicas ocorridas em decorrência da Pandemia.

Como medida preventiva, na maioria dos lugares, as atividades do setor educacional foram suspensas, especificamente, as atividades presenciais, em todos os ambientes escolares em que haveria aglomeração. De modo inesperado e abrupto, estudantes de todos os níveis do ensino tiveram que permanecer em casa (GUSSO et al., 2020; RONDINI; PEDRO; DUARTE, 2020; UNESCO, 2020). No 
Brasil, a solução encontrada para minimizar os problemas relacionados ao fechamento das escolas e Universidades foi a adoção do ensino remoto emergencial (GUSSO et al., 2020; RONDINI; PEDRO; DUARTE, 2020). Essa medida foi disciplinada pelo Ministério da Educação, pela portaria nº 343 , de 17 de março de 2020. Conforme as orientações ministeriais, as atividades presenciais deveriam ser transpostas, por meio de ferramentas digitais, para um modelo de educação remota enquanto durasse a crise sanitária (SARAIVA; TRAVERSINI; LOCKMANN, 2020).

Compreende-se que essa modalidade de ensino é excludente e exacerba as desigualdades já existentes, parcialmente niveladas nos ambientes educacionais (DIAS; PINTO, 2020). Dadas as inequidades socioeconômicas estruturais que prevalecem no país com repercussões na garantia do acesso à educação (GOMES; DUARTE, 2019), entre essas a inequidade digital (DIMAGGIO et al., 2004), é esperado que com a adoção improvisada e de emergência do ensino remoto, a qualidade do acesso às atividades de ensino e aprendizagem e, por conseguinte, o seu aproveitamento tenham se tornado ainda mais desiguais (MARTINS; ALMEIDA, 2020). Isto porque, durante o isolamento doméstico, os estudantes se tornarão inteiramente dependentes das condições de que dispuserem individualmente e privadamente em seus locais de estudo.

Atuando de modo não-verbal, o meio físico da escola tem impacto direto e simbólico sobre seus ocupantes, facilitando ou inibindo comportamentos. As condições ambientais das escolas (acústica, temperatura, insolação, ventilação, luminosidade) podem se refletir em fatores tão diversos quanto a sociabilidade dos usuários, seu desempenho acadêmico e mesmo sua saúde (EALALI, 2003). Essa influência do ambiente da escola se transmuta no ambiente doméstico quando da condição de ensino remoto e de isolamento social que os estudantes enfrentam durante a Pandemia. É a partir desse contexto que se procurou examinar as possíveis relações entre o rendimento acadêmico, que se denomina de “aproveitamento", de estudantes de graduação e pós-graduação e a qualidade de seus ambientes privados de estudo remoto, aqui denominada de "ambiência". Entende-se por rendimento, o aproveitamento qualitativo das atividades de ensino e aprendizagem. Já o conceito de ambiência, operacionalizado neste estudo, segue o proposto pelo Ministério da Saúde que a define como: "tratamento dado ao espaço físico entendido como espaço social, profissional e de relações interpessoais que deve proporcionar atenção acolhedora, resolutiva e humana" (BRASIL, 2010, p.5).

Desse modo, a ambiência vivenciada pelo discente no ensino remoto representa não apenas as condições do ambiente sensorial, mas também está relacionada ao acesso e a qualidade dos equipamentos e serviços que dispõe e utiliza para as práticas de ensino e aprendizagem.

Considerou-se que em tempos de Pandemia e de isolamento social, em tese, o rendimento dos estudantes de graduação e pós-graduação foi em parte determinado pela qualidade da ambiência doméstica de estudo. Para verificar a validade da tese se formulou a seguinte hipótese alternativa verificável: quanto mais favorável a ambiência do aluno de graduação e pós-graduação, maior será seu rendimento. Nesse sentido, este estudo analisa o rendimento de uma amostra de estudantes de graduação e pós-graduação em modalidade de ensino remoto e sua relação com seu ambiente de estudo (ambiência) em período pandêmico da Covid-19 na cidade de Manaus, no final do primeiro semestre de 2020.

Para alcançar tais respostas, foi construída uma ferramenta metodológica que permitiu mensurar de modo objetivo tanto o rendimento quanto a ambiência de cada sujeito da pesquisa. Consideraram-se como covariáveis o gênero do discente e o nível de ensino, isto é, graduação e pós-graduação. A questão de gênero foi inserida no estudo dado que há evidências que o rendimento escolar varia em acordo com esse fator (SILVA et al., 2014). O nível de ensino foi inserido no estudo, uma vez que se espera que o nível de autonomia dos estudantes de pós-graduação seja maior que os dos estudantes de graduação, tanto em termos socioeconômicos com de aprendizagem.

\section{Material e Métodos}

Este estudo se caracteriza como qualitativo e quantitativo, com sabe em amostra não probabilística, composta por 61 estudantes de graduação e pós-graduação (mestrado e doutorado), regularmente matriculados e em atividade, de ambos os sexos, maior e ou igual a 18 anos idade, de universidades públicas, e privadas, em modalidade de ensino remoto emergencial em Manaus/AM.

Para a coleta de dados, foi adotado o método Survey, com a elaboração de um questionário on-line composto por 26 questões, divulgado pela rede de contato dos autores da pesquisa, via aplicativo de mensagens. Após serem recrutados mediante mensagem, os participantes antes de terem acesso às perguntas eram solicitados a confirmar seu consentimento prévio e livremente esclarecido, conforme preceitos éticos da pesquisa com seres humanos. Não foram coletados dados pessoais, mantendo-se o completo anonimato dos participantes.

As perguntas se referem a cada um dos indicadores (Quadro 1) e subindicadores (Quadro 2 e 3), para avaliação da variável independente que corresponde à ambiência e da variável dependente que corresponde ao aproveitamento. Para cada variável foi definido um conjunto de indicadores. Foram ainda analisados os efeitos das covariáveis "nível do curso" (graduação e pós-graduação) e gênero na relação entre as variáveis principais. 
Quadro 1 - Indicadores das variáveis

\begin{tabular}{|c|c|}
\hline $\begin{array}{c}\text { Indicadores, Variável } \\
\text { Independente } \\
\text { (Ambiência) }\end{array}$ & $\begin{array}{c}\text { Indicadores, } \\
\text { Variável Dependente } \\
\text { (Aproveitamento) }\end{array}$ \\
\hline 1. Qualidade do ambiente; & 1. Presença na aula \\
\hline 2. Qualidade de conexão; & $\begin{array}{c}\text { 2. Uso da palavra em cada } \\
\text { aula }\end{array}$ \\
\hline 3. Acesso aos equipamentos; & $\begin{array}{c}\text { 3. Motivo da participação na } \\
\text { aula }\end{array}$ \\
\hline $\begin{array}{c}\text { 4. Familiaridade com as } \\
\text { ferramentas; }\end{array}$ & $\begin{array}{c}\text { 4. Aproveitamento da aula } \\
\text { remota }\end{array}$ \\
\hline $\begin{array}{c}\text { 5. Responsabilidades } \\
\text { adicionais; }\end{array}$ & \\
\hline 6. Estabilidade da rede \\
elétrica.
\end{tabular}

Fonte: Dados da pesquisa.

Após a coleta dos dados primários, em formulário online, foi realizada a tabulação dos pontos de cada participante da pesquisa em planilha eletrônica. Para que fosse possível executar as análises quantitativas, os indicadores qualitativos subjetivos foram convertidos a partir de subindicadores objetivos quantificáveis e metrificados (Quadros 2 e 3). A soma da pontuação, em cada indicador foi ajustada para variar de 0 e 60 pontos. As escalas foram estabelecidas de modo que os maiores valores indicassem a melhor condição de ambiência e de rendimento escolar. Esses valores também foram ajustados para indicar a relevância (ou peso) de cada subindicador para a conformação de uma ambiência favorável ou um melhor aproveitamento.

Quadro 2 - Subindicadores da variável Independente e a respectiva escala

\begin{tabular}{|c|c|}
\hline $\begin{array}{l}\text { Variável independente: Qualidade da ambiência } \\
\text { de estudo }\end{array}$ & Escala \\
\hline \multicolumn{2}{|l|}{ 1. Nível de ruído no ambiente de estudo } \\
\hline a) Ha um bom isolamento acústico & 5 \\
\hline b) Há um nível moderado de ruído & 2 \\
\hline c) Há um nível elevado de ruído & 0 \\
\hline \multicolumn{2}{|l|}{ 2. O ambiente de estudo é compartilhado? } \\
\hline Sim & 0 \\
\hline Não & 1 \\
\hline \multicolumn{2}{|l|}{ 3. Se sim, com quantas pessoas? } \\
\hline $1-2$ Pessoas & 0,5 \\
\hline $2-4$ Pessoas & 0 \\
\hline Acima de 4 & 0 \\
\hline \multicolumn{2}{|l|}{$\begin{array}{l}\text { 3. Qualidade de iluminação no ambiente de } \\
\text { estudo }\end{array}$} \\
\hline Excelente iluminação & 3 \\
\hline Iluminação suficientemente boa & 1 \\
\hline Iluminação pouco adequada & 0 \\
\hline Iluminação inadequada & 0 \\
\hline \multicolumn{2}{|l|}{ 4. Mesas e cadeiras adequadas } \\
\hline São adequadas & 2 \\
\hline Não são adequadas & 0 \\
\hline \multicolumn{2}{|l|}{ Qualidade da conexão } \\
\hline \multicolumn{2}{|l|}{ 5. Internet com queda de sinal } \\
\hline Sim & 1 \\
\hline Não & 2 \\
\hline \multicolumn{2}{|l|}{ 5. Quantas vezes } \\
\hline 0 Quedas de conexão & 3 \\
\hline 1 - 3 Quedas de conexão & 1 \\
\hline
\end{tabular}

Variável independente: Qualidade da ambiência

4 - 6 Quedas de conexão de estudo

ontinuação

Acesso à equipamentos

Marque o Tipo de Equipamento

Celular

Laptop

Desktop

Tablet

Escala

6. Equipamentos compartilhados (ens. Remoto).

Sim

Não

Se sim, com quantas pessoas compartilha.

$1-3$

4-6

Mais de 6 aulas

Familiaridade com ferramentas

7. Aplicativos de aula remota

Hangout

Dropbox

Google classroom

Google meet

$8 \times 8$ meet

Whatsapp

Facebook

Zoom

Skype

Outras atividades/responsabilidades

8. Outras pessoas sob sua responsabilidade

Sim

Não

9. Quantidade de crianças

0 - Crianças

1 - 2 Crianças

2 - Ou mais crianças

10. Quantidade de adolescente

0 - Adolescentes

1 - 2 Adolescentes

3 - Ou mais adolescentes

11. Quantidade de idosos

0 Idosos

1 - 2 Idosos

3 ou mais idosos

11. Trabalho simultâneo

Sim

Não

12. Negociação de hora

Sim

Não

13. Regime de trabalho

Integral

Parcial

Negociação de horas

Estabilidade rede elétrica

14. Estabilidades de rede elétrica

Excelente

Bom

Regular

Ruim

15. Interrupção da rede elétrica

0 - Quedas

1 - 3 Quedas

4 - 6 Quedas de conexão

Total

Fonte: Dados da pesquisa.

0

\begin{tabular}{|c|c|}
\hline & \\
\hline & 0,5 \\
\hline & 3 \\
\hline & 0,5 \\
\hline & 0 \\
\hline & 1 \\
\hline & 2 \\
\hline & 1 \\
\hline
\end{tabular}

(1)

\begin{tabular}{|l|l|} 
& 0 \\
\hline & 1 \\
\hline & 5 \\
\hline & 0 \\
\hline & 1 \\
\hline & 0 \\
\hline
\end{tabular}


Quadro 3 - Subindicadores da variável Dependente e a respectiva escala

\begin{tabular}{|l|l|}
\hline $\begin{array}{l}\text { Variável Dependente: Aproveitamento dos } \\
\text { estudantes }\end{array}$ & Escala \\
\hline 16. Quantas vezes não participou da aula & 5 \\
\hline 0 - Não perdi aulas & 3 \\
\hline 1 - 3 aulas & 2 \\
\hline 4 - 6 aulas & 0 \\
\hline Mais de 6 aulas & 5 \\
\hline 17. Uso da palavra a cada encontro & 4 \\
\hline Em todos os encontros & 2 \\
\hline Na maioria das vezes & 1 \\
\hline Apenas algumas vezes & 1 \\
\hline Poucas vezes & 6 \\
\hline Raramente & 2 \\
\hline 18. Participou ativamente das aulas porque: & 1 \\
\hline Ajudavam a detectar e superar minhas dúvidas & 1 \\
\hline Sentiria mal se não fizesse & 4 \\
\hline Não me sentia interesse & 3 \\
\hline Porque seria reprovado na disciplina & 10 \\
\hline Boa compreensão & 5 \\
\hline Deixa feliz, satisfeito... & 2 \\
\hline 19. Aproveitamento nas aulas remotas & $\mathbf{6 0}$ \\
\hline $\begin{array}{l}\text { Tenho conseguido acompanhar, compreender e } \\
\text { assimilar. }\end{array}$ & \begin{tabular}{l}
$\mid l$ \\
\hline Acompanhar compreender quase todos os conteúdos
\end{tabular} \\
\hline $\begin{array}{l}\text { Não tenho sido capaz de acompanhar, compreender } \\
\text { uma grande parte dos conteúdos. }\end{array}$ \\
\hline $\begin{array}{l}\text { Não consigo acompanhar, compreender a maioria dos } \\
\text { conteúdos. }\end{array}$ & 5 \\
\hline Total & 2 \\
\hline
\end{tabular}

Fonte: Dados da pesquisa.

Após a tabulação da pontuação de cada participante, via os critérios estabelecidos acima, os dados foram analisados com apoio do programa estatístico Past, versão 4.03 para análise descritiva. Nesse programa, foram gerados gráficos (dispersão e box plot) e quadros que correspondem às análises das covariáveis gênero e nível de curso (mestrado e doutorado). A relação entre as variáveis principais foi explorada mediante a técnica de análise de regressão linear simples e o efeito da covariáveis foi verificado pelo teste de modelo de regressão linear múltipla.

Considerando-se o conjunto da amostra e a resposta de todos os estudantes participantes da pesquisa, avaliouse o desempenho das duas variáveis para cada indicador. Calculou-se o percentual de desempenho de cada indicador dividindo-se a soma da pontuação do item pelo máximo de pontuação acumulada que corresponde ao valor máximo do indicador vezes o tamanho de amostra. Essa avaliação serviu para identificar como variou cada componente da ambiência e do rendimento no conjunto das autoavaliações, o que serviu para indicar a condição mediana da experiência dos estudantes da cidade que foram submetidos a situação de ensino remoto durante o isolamento social.

\section{Resultados e Discussão}

Os participantes desta pesquisa eram estudantes de graduação e de pós-graduação de universidades públicas e privadas da cidade de Manaus. Da amostra coletada, embora se tenha buscado a equidade na representatividade, as mulheres corresponderam a ligeira maioria. Quanto à covariável 'nível de curso', a maioria era formada por estudantes de graduação e o restante de pós-graduação (mestrado e doutorado) (Quadro 4). As mulheres corresponderam a $66 \%$ dos estudantes de graduação e $46 \%$ de pós-graduação.

Quadro 4 - Dados gerais da amostra

\begin{tabular}{|l|c|c|}
\hline Covariáveis & $\mathbf{N}^{\mathbf{0}}$ Participantes & \% \\
\hline Gênero: & & 44 \\
\hline Masculino & 27 & 56 \\
\hline Feminino & 34 & \\
\hline Nível de curso: & & 57 \\
\hline Graduação & 35 & 43 \\
\hline Pós-graduação & 26 & \\
\hline
\end{tabular}

Fonte: Dados da pesquisa.

Como esperado o grupo de estudantes de graduação apresentou um média de idade menor com maior frequência na faixa de idade entre 18 e 23 anos. O grupo de pósgraduandos apresentou as faixas de 24 a 34 anos com as de maior ocorrência (Quadro 5). Houve boa dispersão dos dados em ambos os grupos, indicando que o estudo contou com uma amostra representativa e não tendenciosa em termos de idade dos estudantes.

Quadro 5 - Distribuição dos estudantes participantes por faixa etária

\begin{tabular}{|l|c|c|c|}
\hline $\begin{array}{c}\text { Faixa Etária } \\
\text { (Anos) }\end{array}$ & Graduação & $\begin{array}{c}\text { Pós- } \\
\text { graduação }\end{array}$ & Total Geral \\
\hline 18 a 23 & 16 & 1 & 17 \\
\hline 24 a 28 & 4 & 4 & 8 \\
\hline 29 a 34 & 7 & 6 & 14 \\
\hline 35 a 40 & 1 & 7 & 8 \\
\hline 41 a 45 & 3 & 5 & 8 \\
\hline 46 a 50 & 1 & 2 & 3 \\
\hline >51 & 3 & 1 & 4 \\
\hline Total Geral & $\mathbf{3 5}$ & $\mathbf{2 6}$ & $\mathbf{6 1}$ \\
\hline
\end{tabular}

Fonte: Dados da pesquisa.

Entre as componentes que compuseram à avaliação da ambiência, os piores desempenhos $(<50 \%)$ foram obtidos nos indicadores do compartilhamento do ambiente de estudo e dos equipamentos, do nível de ruído, da interrupção do sinal de internet e do trabalho simultâneo (Figura 1). Essa é uma clara indicação de que a qualidade da ambiência pode variar amplamente entre os estudantes em razão das novas condições impostas pela Pandemia. No isolamento doméstico, a oferta das condições objetivas que garantam a qualidade do ambiente de aprendizagem passa a depender, exclusivamente, da capacidade de cada indivíduo ou grupo doméstico, gerando desigualdades ou aprofundando as já existentes. 
Figura 1 - Desempenho geral dos indicadores de ambiência do ensino remoto

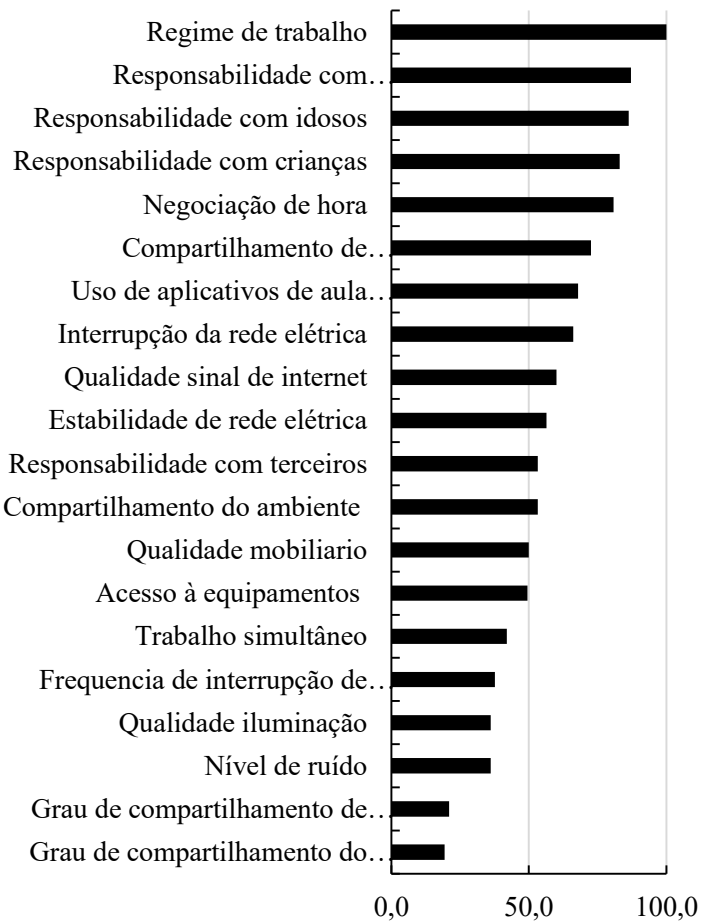

Fonte: Dados da pesquisa.

Quanto ao rendimento, o pior desempenho $(<50 \%)$ foi observado na componente "uso da palavra". Esse "silenciamento" durante as aulas síncronas e assíncronas agravados pela condição de distanciamento e deslocamento físico pode impor barreiras ao processo de ensino e aprendizagem, uma vez que denota a ausência de interatividade e diálogo entre os estudantes e destes com o docente (SILVA, 2004). Nas outras três dimensões da autoavaliação, os estudantes entrevistados consideraram seus desempenhos muito favoravelmente (>75\%) (Figura 2).

Figura 2 - Desempenho dos indicadores de rendimento dos estudantes

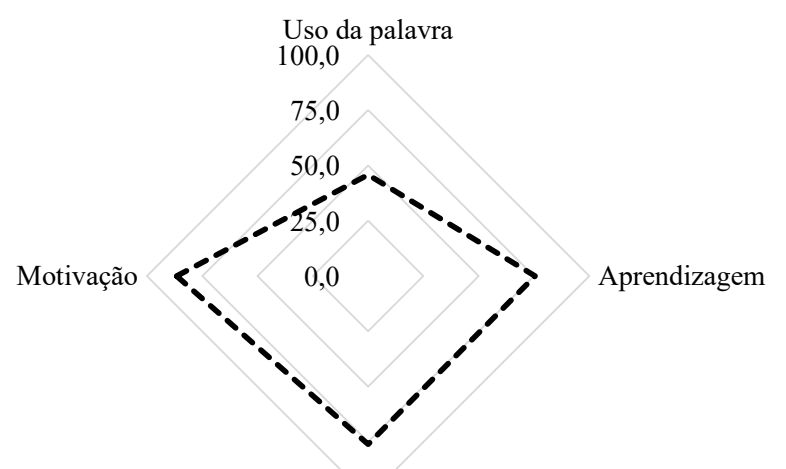

Participação

Fonte: Dados da pesquisa.

De um modo geral, tomando-se a amostra como um todo, ou seja, ambos gêneros e níveis do curso, observou-se que há uma correlação positiva entre ambiência e o rendimento escolar, sendo que a ambiência é capaz de explicar até
$15,4 \%$ da variação no rendimento (Figura 3). Apesar de ser considerada uma correlação fraca, esse valor foi significativo estatisticamente $(\mathrm{p}<<0,01)$. Para cada incremento de uma unidade na qualidade da ambiência, a melhora no rendimento pode ser de 0,20 a 0,69 pontos (Quadro 5).

Figura 3 - Análise de regressão da amostra total

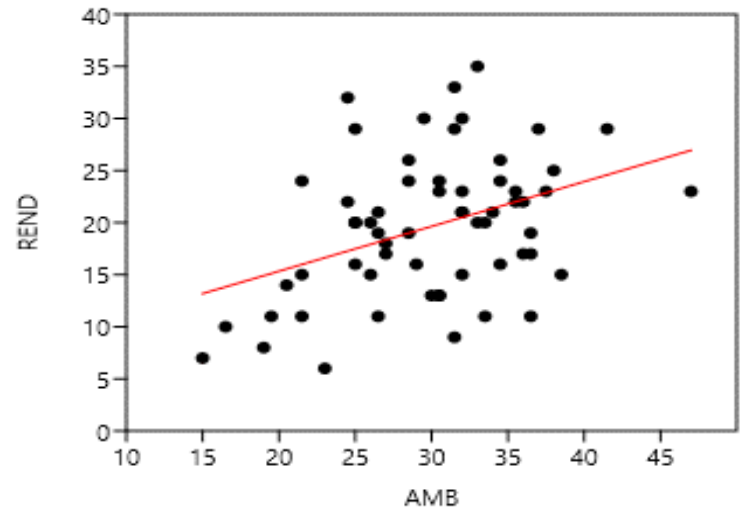

RENDm: Rendimento masculino; RENDf: Rendimento feminino;AMBm: Ambiência masculino; AMBf: Ambiência feminino.

Fonte: Dados da pesquisa.

Quadro 6 - Análise da regressão AMB - REND

\begin{tabular}{|c|c|}
\hline \multicolumn{2}{|c|}{ Regressão linear simples: AMB-REND } \\
\hline Inclinação a: & 0,43 \\
\hline $\mathrm{t}:$ & 3,28 \\
\hline Intercepto b: & 6,74 \\
\hline Erro padrão de a: & 0,13 \\
\hline P (inclinação): & 0,002 \\
\hline Erro padrão de b: & 4,0015 \\
\hline Intervalos de confiança 95\% bootstrap (N=1999): \\
\hline Inclinação a: & $(0,20,0,69)$ \\
\hline Intercepto b: & $(-1,65,13,44)$ \\
\hline \multicolumn{2}{|c|}{ Correlação } \\
\hline r: & 0,39 \\
\hline r2: & 0,15 \\
\hline t: & 3,28 \\
\hline p (não corr.): & 0,0017 \\
\hline Permutação p: & 0,0013 \\
\hline
\end{tabular}

Fonte: Dados da pesquisa.

Averiguando-se as variáveis dependente e independente em relação à covariável gênero, percebeu-se que a distribuição empírica dos dados de ambas as variáveis não se mostrou muito diferente na comparação entre os gêneros, como se observa nos gráficos Boxplot (Figura 4). No entanto, a média e a mediana do rendimento do gênero masculino (RENDm) foram menores que as do rendimento feminino (RENDf), sendo o contrário observado em relação à variável independente Ambiência. Apenas no caso da amostra feminina é que foi detectado um valor discrepante para ambiência, acima do nível superior (Quadro 7). 
Figura 4 - Análise da covariável gênero, das variáveis independentes e dependentes
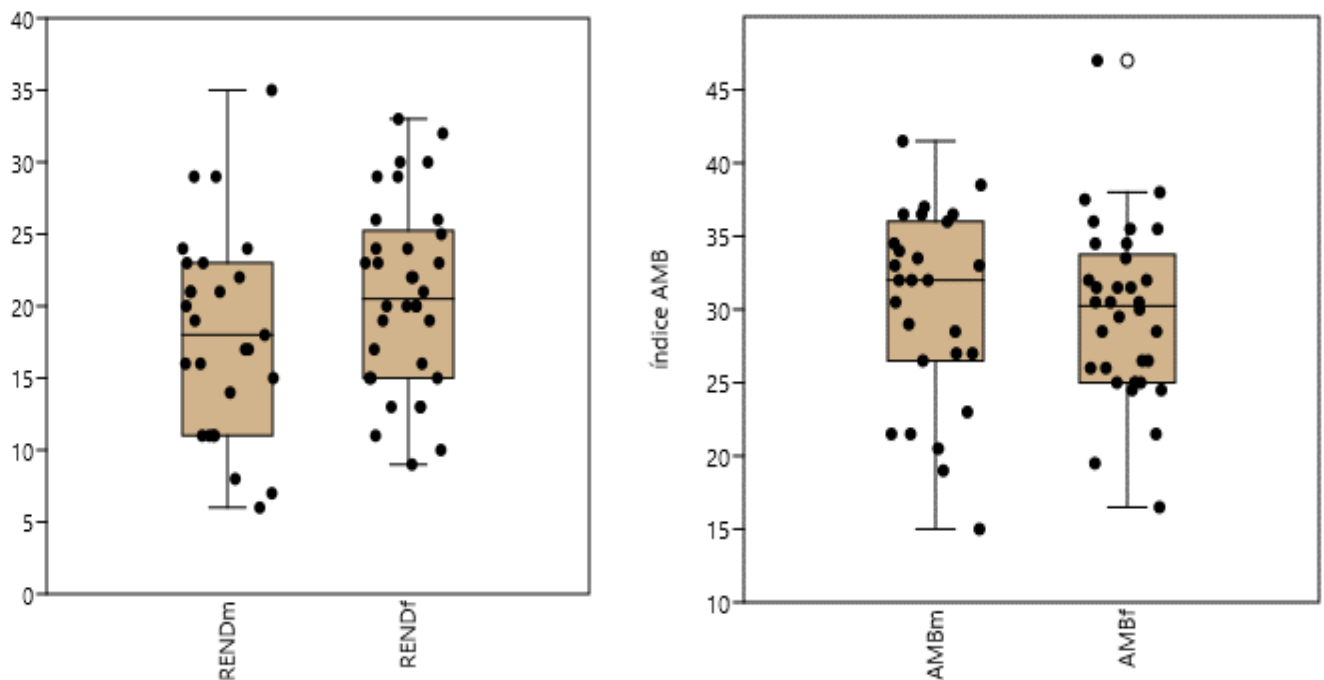

RENDm: Rendimento masculino; RENDf: Rendimento feminino; AMBm: Ambiência masculino; AMBf: Ambiência feminino Fonte: Dados da pesquisa.

Quadro 7 - Estatísticas univariadas das amostras por gênero

\begin{tabular}{|l|c|c|c|c|}
\hline & RENDm & RENDf & AMBm & AMBf \\
\hline $\begin{array}{l}\text { Tamanho de } \\
\text { amostra }\end{array}$ & 27 & 34 & 27 & 34 \\
\hline $\begin{array}{l}\text { Valor } \\
\text { Mínimo }\end{array}$ & 6 & 9 & 15 & 16,5 \\
\hline $\begin{array}{l}\text { Valor } \\
\text { Máximo }\end{array}$ & 35 & 33 & 41,5 & 47 \\
\hline Média & 18,11 & 20,79 & 30,2 & 29,71 \\
\hline Erro padrão & 1,36 & 1,1 & 1,28 & 1,01 \\
\hline Variância & 49,87 & 41,5 & 44,5 & 35,08 \\
\hline $\begin{array}{l}\text { Desvio } \\
\text { padrão }\end{array}$ & 7,06 & 6,44 & 6,67 & 5,92 \\
\hline Mediana & 18 & 20,5 & 32 & 30,25 \\
\hline $\mathbf{1}^{\mathbf{0} Q u a r t i l}$ & 11 & 15 & 26,5 & 25 \\
\hline $3^{\mathbf{0}}$ Quartil & 23 & 25,25 & 36 & 33,75 \\
\hline Skewness & 0,29 & 0,04 & $-0,58$ & 0,37 \\
\hline Kurtosis & $-0,04$ & $-0,74$ & $-0,38$ & 1,22 \\
\hline $\begin{array}{l}\text { Média } \\
\text { geométrica }\end{array}$ & 16,65 & 19,74 & 29,39 & 29,12 \\
\hline $\begin{array}{l}\text { Coeficiente } \\
\text { de variação }\end{array}$ & 38,99 & 30,98 & 22,09 & 19,94 \\
\hline
\end{tabular}

RENDm: Rendimento masculino; RENDf: Rendimento feminino;AMBm: Ambiência masculino; AMBf: Ambiência feminino.

Fonte: Dados da pesquisa.

Para avaliar o efeito da covariável gênero sobre as duas variáveis principais, foram observados os valores de $p$ para o teste $t$ das médias. No caso da variável independente, Ambiência, teste indicou não haver diferença entres os gêneros (AMBm vs. AMBf) ( $p>>0,05)$. Já com relação ao rendimento, a diferença entre as médias chega a 2,68 pontos em média, a mais para a amostra feminina. A probabilidade de rejeição da hipótese nula é bem menor $(\mathrm{p}=0,113)$, porém ainda não significativa. No entanto, deve-se considerar que nessa análise não está sendo isolado o efeito do nível do curso, como nas análises que se seguem (Quadros 8 e 9).
Quadro 8 - Testes das medias iguais dos gráficos 2 variáveis independentes

\begin{tabular}{|l|c|c|}
\hline \multicolumn{1}{|c|}{ Parâmetros } & AMBm & AMBf \\
\hline Tamanho da amostra & 27 & 34 \\
\hline Média & 30,2 & 29,71 \\
\hline 95\% confiança & $(27,5632,84)$ & $(27,6431,77)$ \\
\hline Variância: & 44,5 & 35,08 \\
\hline Diferença entre as médias & 0,498 & \\
\hline $\begin{array}{l}\text { 95\% intervalo de confiança } \\
\text { (paramétrico): }\end{array}$ & $(-2,733,73)$ & \\
\hline $\begin{array}{l}\text { 95\% intervalo de conf } \\
\text { (bootstrap): }\end{array}$ & $(-2,673,73)$ & \\
\hline t & 0,308 & $\begin{array}{c}\text { p (mesma } \\
\text { média): } 0,7589\end{array}$ \\
\hline t variância desigual: & 0,304 & $\begin{array}{c}\text { p (mesma } \\
\text { média): }\end{array}$ \\
\hline Monte Carlo permuta: & $\begin{array}{c}\text { p (mesma } \\
\text { média): }\end{array}$ & 0,7752 \\
\hline
\end{tabular}

AMBm: Ambiência masculino; AMBf: Ambiência feminino.

Fonte: Dados da pesquisa.

Quadro 9 - Testes das medias iguais dos gráficos 2, variáveis dependentes

\begin{tabular}{|l|c|c|}
\hline Parâmetros & RENDm & RENDf \\
\hline Tamanho da amostra & 27 & 34 \\
\hline Média & 18,11 & 20,79 \\
\hline 95\% confiança & $(15,3220,90)$ & $(18,5623,04)$ \\
\hline Variância & 49,87 & 41,5 \\
\hline Diferença entre médias & 2,68 & \\
\hline $\begin{array}{l}\text { 95\% Intervalo de } \\
\text { Confiança (paramétrico) }\end{array}$ & $(-0,786,15)$ & \\
\hline $\begin{array}{l}\text { 95\% intervalo de } \\
\text { confiança (bootstrap): }\end{array}$ & $(-0,625,99)$ & \\
\hline & 1,548 & $\begin{array}{c}\text { p (mesma } \\
\text { média): } 0,1315\end{array}$ \\
\hline t & 1,532 & 0,1294 \\
\hline t variância desigual: & $\begin{array}{c}\text { p (mesma } \\
\text { média): }\end{array}$ & $\begin{array}{c}\mathrm{p} \text { (mesma } \\
\text { média): }\end{array}$ \\
\hline Monte Carlo permuta: & &
\end{tabular}

RENDm: Rendimento masculino; RENDf: Rendimento feminino.

Fonte: Dados da pesquisa. 
Dada a indicação de um provável efeito de gênero, a amostra foi segmentada por essa covariável e os resultados das duas análises de regressão linear simples foram analisados. Para a subamostra masculina, a relação entre a ambiência e rendimento foi significativa $(p=0,005)$, sendo o contrário observado na amostra feminina. Os coeficientes de regressão e de correlação também indicaram claramente a influência da covariável gênero na relação entre as variáveis principais (Figura 5). Apenas para o gênero masculino a correlação entre ambiência e rendimento foi significativa $(p=0,005)$, sendo que esta variável independente consegue explicar até $27 \%$ da variável dependente. No caso da amostra feminina, os resultados indicam que o rendimento das estudantes não é influenciado pela ambiência (Quadro 10).
Figura 5 - Análise de regressão linear simples da covariável gênero

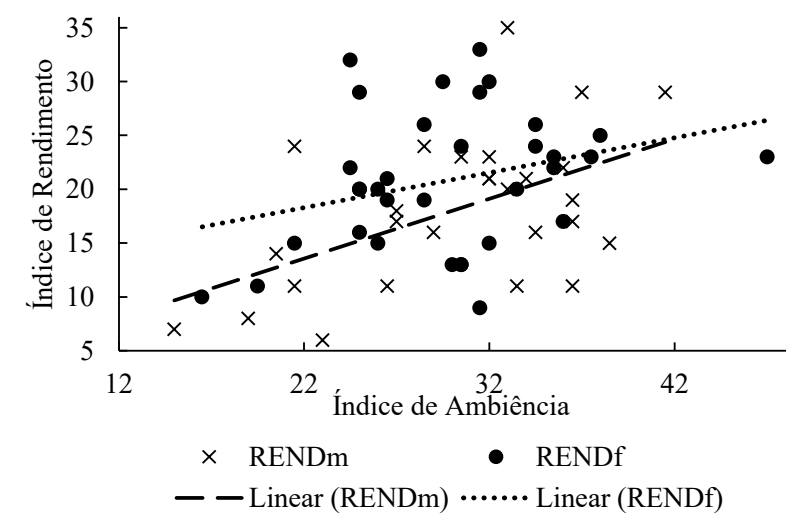

RENDm: Rendimento masculino; RENDf: Rendimento feminino.

Fonte: Dados da pesquisa.

Quadro 10 - Análise de regressão linear com covariável gênero

\begin{tabular}{|c|c|c|c|c|}
\hline \multicolumn{5}{|c|}{ Regressão Linear Simples: AMBm-RENDm } \\
\hline Inclinação a: & 0,55473 & Erro padrão a: & 0,18032 & 0,005 \\
\hline & $\mathrm{t}:$ & 3,0764 & $\mathrm{p}$ (Inclinação): & \\
\hline Intercepto b: & 1,3564 & Erro padrão b: & 5,5727 & \\
\hline \multicolumn{5}{|c|}{ Regressão Linear Simples: AMBf-RENDf } \\
\hline Inclinação a: & 0,32391 & Erro padrão a: & 0,18356 & 0,087 \\
\hline Intercepto b: & $\mathrm{t}:$ & 1,7646 & $\mathrm{p}$ (Inclinação): & \\
\hline
\end{tabular}

AMBm: Ambiência masculino;RENDm: Rendimento masculino; AMBf: Ambiência feminino; RENDf: Rendimento feminino.

Fonte: Dados da pesquisa.

Já em relação à covariável nível de curso, percebe-se que as distribuições empíricas dos dados se mostraram bastante diferentes na comparação entre o nível de graduação $(\mathrm{g})$ e de pós-graduação (pg), como se observa nos gráficos Boxplot (Figura 6). A média e mediana do rendimento escolar é bem superior na subamostra de pós-graduação, o que não se observa para a variável independente ambiência. Em ambas as variáveis, a subamostra de pós-graduação apresenta uma menor dispersão dos valores indicando que nesse nível os discentes experimentam condições menos desiguais (Quadro 11).

Figura 6 - Análise de covariável nível de curso, das variáveis independentes e dependentes
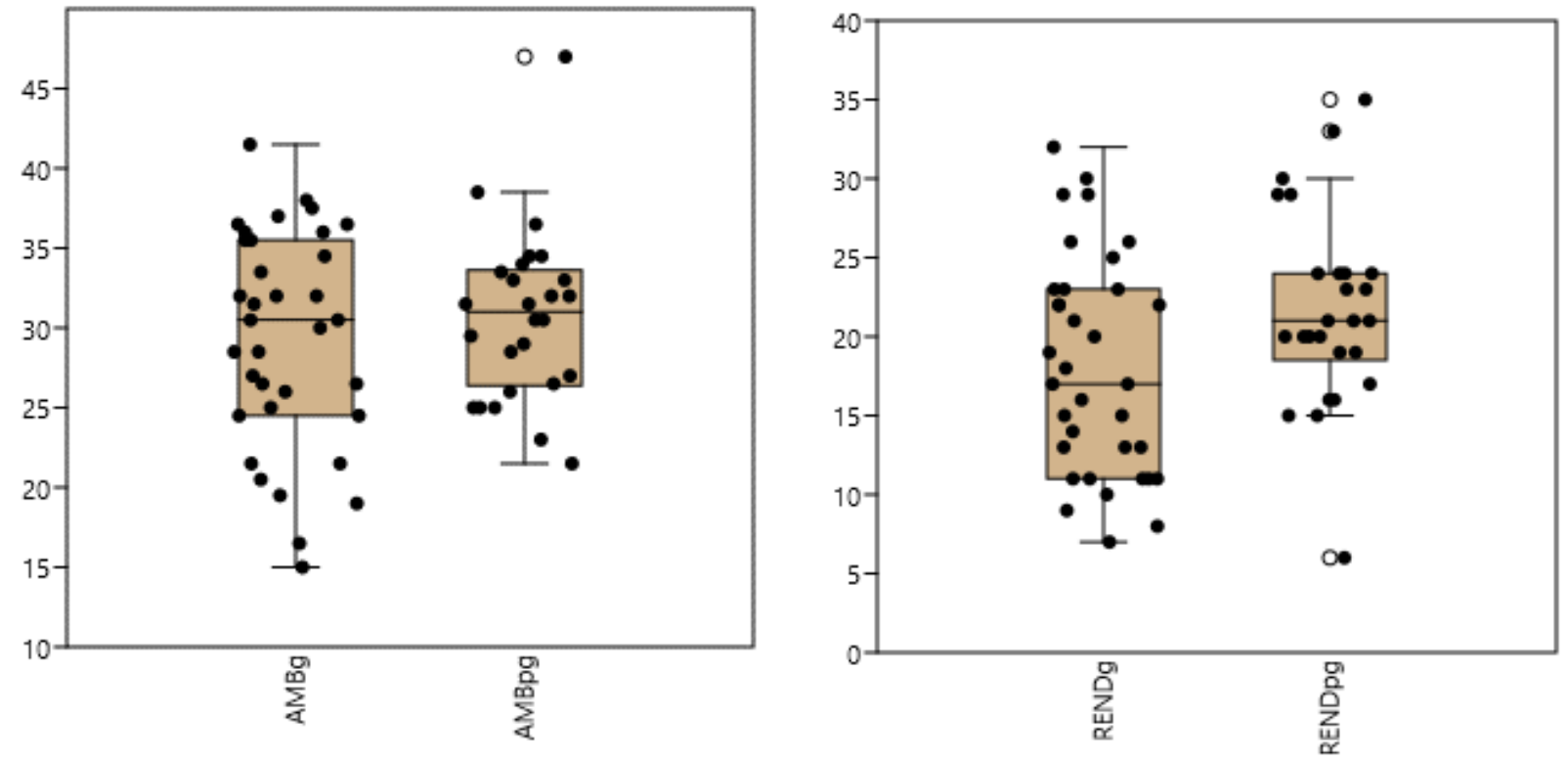

AMBg: Ambiência graduação; AMBf: Ambiência pós-graduação; RENDg: Rendimento graduação; RENDpg: Rendimento pós-graduação.

Fonte: Dados da pesquisa. 
Quadro 11 - Estatísticas univariadas das amostras por nível de ensino

\begin{tabular}{|c|c|c|c|c|}
\hline & RENDg & RENDpg & AMBg & AMBpg \\
\hline N & 35 & 26 & 35 & 26 \\
\hline Mínimo & 7 & 6 & 15 & 21,5 \\
\hline Máximo & 32 & 35 & 41,5 & 47 \\
\hline Média & 18,06 & 21,69 & 29,34 & 30,71 \\
\hline $\begin{array}{c}\text { Erro } \\
\text { padrão }\end{array}$ & 1,17 & 1,21 & 1,15 & 1,06 \\
\hline Variância & 47,7 & 38,22 & 45,98 & 29,08 \\
\hline $\begin{array}{c}\text { Desvio } \\
\text { padrão }\end{array}$ & 6,91 & 6,18 & 6,78 & 5,39 \\
\hline Mediana & 17 & 21 & 30,5 & 31 \\
\hline $\mathbf{1}^{\mathbf{0}}$ quartil & 11 & 18,5 & 24,5 & 26,37 \\
\hline $\mathbf{3}^{\mathbf{0}}$ Quartil & 23 & 24 & 35,5 & 33,62 \\
\hline Skewness & 0,29 & 0,04 & $-0,37$ & 0,87 \\
\hline $\begin{array}{c}\text { Kurtosis } \\
\text { Média } \\
\text { Geométrica }\end{array}$ & $-0,96$ & 0,98 & $-0,72$ & 2,06 \\
\hline $\begin{array}{c}\text { Coeficiente } \\
\text { de } \\
\text { Variação }\end{array}$ & 38,72 & 20,68 & 28,5 & 30,28 \\
\hline RED Ren & 28,5 & 23,11 & 17,56 \\
\hline
\end{tabular}

RENDg: Rendimento graduação; RENDpg: Rendimento pós-graduação; AMBg: Ambiência graduação; AMBf: Ambiência pós-graduação.

Fonte: Dados da pesquisa.

Para avaliar o efeito da covariável "Nível do curso" sobre as duas variáveis principais, foram observados os valores de $\mathrm{p}$ para o teste $t$ das médias. No caso da variável independente, ambiência, o teste indicou não haver diferença entres os níveis (AMBg vs. AMBpg; $p>>0,05$ ), indicando que a amostra cobriu uniformemente ambos os níveis. Já com relação ao rendimento, a diferença entre as médias chega a 3,63 pontos em média, a mais para a amostra da pósgraduação. A probabilidade de rejeição da hipótese menor que $0.05(\mathrm{p}=0,0378)$, sendo a diferença, portanto, significativa. Isso indica que mesmo enfrentando condições consideradas semelhantes, alunos de pós-graduação tendem a avaliar seu desempenho mais favoravelmente do que alunos de graduação (Quadros 12 e 13).

Quadro 12 - Teste das médias iguais das amostras por nível de ensino para a variável independente Ambiência

\begin{tabular}{|l|c|c|}
\hline & AMBg & AMBpg \\
\hline Tamanho da amostra & 35 & 26 \\
\hline Média & 29,34 & 30,71 \\
\hline $\mathbf{9 5 \%}$ confiança & $(27,0131,67)$ & $(28,5332,89)$ \\
\hline Variância & 45,98 & 29,08 \\
\hline Diferença entre as médias & 1,37 & \\
\hline $\begin{array}{l}\text { 95\% intervalo de confiança } \\
\text { (paramétrico): }\end{array}$ & $(-1,864,60)$ & \\
\hline $\begin{array}{l}\text { 95\% intervalo de confiança } \\
\text { (bootstrap): }\end{array}$ & $(-1,654,33)$ & \\
\hline t & 0,8484 & $\begin{array}{c}\mathrm{p}(\text { mesma } \\
\text { média): } 0,3996\end{array}$ \\
\hline mesma variância t & 0,8776 & $\begin{array}{c}\mathrm{p}(\text { mesma } \\
\text { média): } 0,3837\end{array}$ \\
\hline Monte Carlo permuta: & $\begin{array}{c}\mathrm{p} \text { (mesma } \\
\text { média): }\end{array}$ & 0,4096 \\
\hline
\end{tabular}

AMBg: Ambiência graduação; AMBf: Ambiência pós-graduação.

Fonte: Dados da pesquisa.
Quadro 13 - Teste das médias iguais das amostras por nível de ensino para a variável dependente Rendimento

\begin{tabular}{|l|c|c|}
\hline & RENDg & RENDpg \\
\hline Tamanho da amostra & 35 & 26 \\
\hline Média & 18,06 & 21,69 \\
\hline 95\% confiança & $(15,6820,43)$ & $(19,1924,19)$ \\
\hline Variância & 47,7 & 38,22 \\
\hline $\begin{array}{l}\text { Diferença entre as } \\
\text { médias95\% intervalo de } \\
\text { confiança (paramétrico): }\end{array}$ & 3,6352 & \\
\hline $\begin{array}{l}\text { 95\% intervalo de confiança } \\
\text { (bootstrap): }\end{array}$ & $(0,217,06)$ & \\
\hline t & $(0,446,84)$ & \\
\hline $\begin{array}{l}\text { mesma variância tMonte } \\
\text { Carlo permuta: }\end{array}$ & 2,1243 & $\begin{array}{c}\mathrm{p}(\mathrm{mesma} \\
\text { média): } 0,0378\end{array}$ \\
\hline Tamanho da amostra & 2,1597 & $\begin{array}{c}\mathrm{p}(\mathrm{mesma} \\
\text { média): } 0,035\end{array}$ \\
\hline Média & $\begin{array}{c}\mathrm{p}(\mathrm{mesma} \\
\text { média): }\end{array}$ & 0,0377 \\
\hline
\end{tabular}

RENDg: Rendimento graduação; RENDpg: Rendimento pós-graduação.

Fonte: Dados da pesquisa.

Dada a indicação de um provável efeito do nível de curso, a amostra foi segmentada por essa covariável e os resultados das duas análises de regressão linear simples foram analisados. Para a subamostra de Graduação, a relação entre a ambiência e rendimento foi altamente significativa $(\mathrm{p}=0,0016)$, sendo o contrário observado na amostra da pós-graduação $(\mathrm{p}=0,541)$ (Quadro 14). Os coeficientes de regressão também indicam claramente a influência da covariável "Nível de curso" na relação entre as variáveis principais (Figura 7). Apenas para subamostra de graduação a correlação entre ambiência e rendimento foi significativa $(\mathrm{p}=0,0016)$, sendo a variável independente a que consegue explicar até $26 \%$ da variável dependente.

Figura 7 - Análise de regressão linear simples da covariável nível de curso

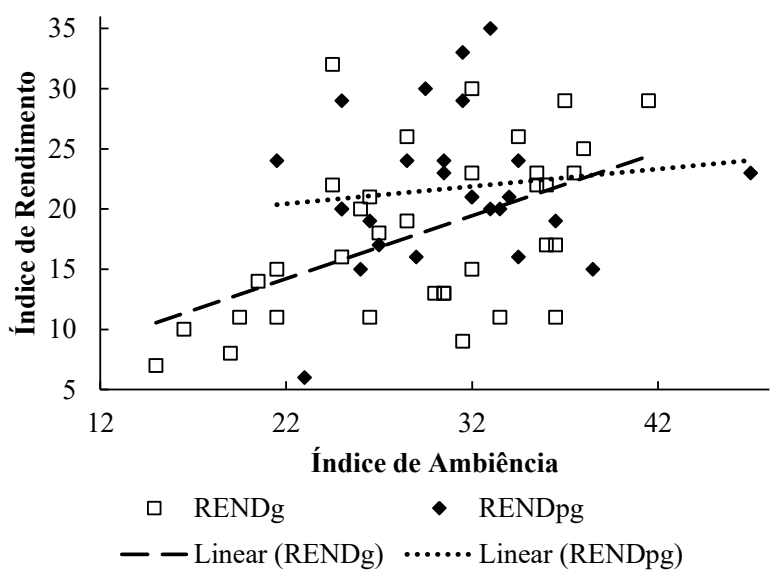

RENDg: Rendimento graduação; RENDpg: Rendimento pós-graduação. Fonte: Dados da pesquisa. 
Quadro 14 - Análise de regressão linear com covariável nível do curso

\begin{tabular}{|c|c|c|c|c|}
\hline \multicolumn{5}{|c|}{ Regressão Linear Simples: AMBg-RENDg } \\
\hline Inclinação a: & 0,52 & Erro padrão a: & 0,15 & 0,0016 \\
\hline & $\mathrm{t}:$ & 3,4384 & $\mathrm{p}$ (Inclinação): & \multicolumn{2}{c|}{4,58} \\
\hline Intercepto b: & 2,71 & Erro padrão b: & 0,23 & \\
\hline \multicolumn{7}{|c|}{ Regressão Linear Simples: AMBpg-RENDpg } \\
\hline Inclinação a: & 0,14 & Erro padrão a: & $\mathrm{p}$ (Inclinação): & 0,5409 \\
\hline & $\mathrm{t}:$ & 0,6202 & 7,23 & \\
\hline
\end{tabular}

AMBg: Ambiência graduação; AMBf: Ambiência pós-graduação; RENDg: Rendimento graduação; RENDpg: Rendimento pós-graduação.

Fonte: Dados da pesquisa.

Para se avaliar o efeito conjunto das duas covariáveis sobre a relação das variáveis principais, analisaram-se os resultados de uma análise de regressão linear múltipla. Para isso, as covariáveis foram inseridas no modelo como variáveis binárias. O modelo completo consegue explicar $23 \%$ da variável dependente, o rendimento, tendo sido altamente significativo $(\mathrm{p}<<0,001)$. Isso indica que outras variáveis, provavelmente, também interferem no rendimento e que não estão associadas com a ambiência. Todas as variáveis do modelo foram significativas. No caso de gênero, houve indicação de que o rendimento das mulheres é melhor que a dos homens para um mesmo nível de qualidade de ambiência. De modo semelhante, o rendimento dos estudantes de pósgraduação é superior se comparado com os de graduação em um mesmo nível de qualidade de ambiência. Quando o efeito da ambiência é controlado pelas covariáveis essa variável independente consegue explicar até $15 \%$ do rendimento escolar (Quadro 15).

Quadro 15 - Análise de regressão linear múltipla das duas covariáveis sobre a relação das variáveis principais

\begin{tabular}{|c|c|c|c|c|c|}
\hline \multicolumn{6}{|c|}{ Variável Dependente: Rendimento } \\
\hline $\mathrm{N}$ & 61 & & & & \\
\hline R múltiplo & 0,52 & & & & \\
\hline $\mathrm{R}^{2}$ múltiplo & 0,27 & & & & \\
\hline $\begin{array}{l}\mathrm{R}^{2} \text { múltiplo } \\
\text { ajustado }\end{array}$ & 0,23 & & & & \\
\hline \multicolumn{6}{|l|}{ ANOVA } \\
\hline $\mathrm{F}$ & 7 & & & & \\
\hline gl1, gl2 & 3,57 & & & & \\
\hline \multirow[t]{2}{*}{$\mathrm{p}$} & 0,0004 & & & & \\
\hline & Coeficiente & $\begin{array}{c}\text { Erro } \\
\text { padrão }\end{array}$ & $\mathrm{t}$ & $\mathrm{p}$ & $\mathrm{R}^{2}$ \\
\hline Constante & 3,86 & 3,94 & 0,98 & 0,331 & \\
\hline $\begin{array}{l}\text { Gênero } \\
\text { (Masculino = } \\
0 ; \text { Feminino } \\
=1 \text { ) }\end{array}$ & 3,49 & 1,56 & 2,24 & 0,029 & 0,04 \\
\hline $\begin{array}{l}\text { Nível do Curso } \\
\text { (Graduação } \\
=0 \text {; Pós- } \\
\text { graduação = 1) }\end{array}$ & 3,66 & 1,57 & 2,32 & 0,024 & 0,07 \\
\hline
\end{tabular}

Fonte: Dados da pesquisa.

Os resultados indicaram que existe uma superioridade do rendimento acadêmico do gênero feminino em relação ao gênero masculino, independente da qualidade da ambiência.
Esse achado corrobora com resultados de diversos outros estudos que indicam haver vantagem feminina generalizada nas notas escolares para a maioria das disciplinas dos cursos em diversos contextos (VOYER; VOYER, 2014).

Senkevics e Carvalho (2016) argumentam que meninas se mostraram capazes de se apropriar de suas realidades para potencializar suas capacidades e perspectivas, ainda que essas pouco se efetivassem na prática àquela altura de seus percursos de vida. Não por acaso, muitas delas se mostraram críticas às situações vividas. Em seus sonhos para o futuro talvez a principal crítica ao seu próprio cotidiano - orientavam seu esforço, disciplina e desempenho na escola, permitindo-as vislumbrar novos horizontes de vida.

No Brasil, a avaliação nacional da qualidade do ensino é realizada com alunos do $4^{\circ}$ e $9^{\circ}$ ano do Ensino Fundamental. O Ministério da Educação (MEC) utiliza os resultados para implementar as políticas públicas necessárias à melhoria de todo o sistema. A proficiência exigida para os estudantes de todo o Brasil é o nível 4 ou superior, nas disciplinas de Língua Portuguesa e Matemática. No Amazonas, considerando-se os resultados da Prova Brasil, as meninas apresentaram melhor proficiência em Língua Portuguesa $(36 \%)$ do que os meninos (21\%). Em Matemática, a relação se inverte, meninos (30\%) alcançam resultado médio superior ao das meninas (21\%) (ATLAS ODS AMAZONAS, 2020).

Asanov et al. (2021) conduziram uma pesquisa de resposta rápida por telefone no Equador com mais de 1.500 alunos do Ensino Médio de 14 a 18 anos para saber como os alunos passam o tempo durante o período de quarentena. As diferenças de gênero no aprendizado à distância são pequenas, com as mulheres fazendo um pouco mais de trabalho escolar. Os estudantes do sexo feminino também realizaram mais tarefas domésticas do que os do sexo masculino, enquanto os estudantes do sexo masculino têm maior probabilidade de trabalhar do que as do sexo feminino. Os alunos do último ano ( $12^{\mathrm{a}}$ série) passam mais tempo estudando do que as séries mais novas.

Blazquez-Merino et al. (2019) utilizaram uma combinação de atividades teóricas e tarefas práticas por meio das quais as habilidades cognitivas e motoras foram medidas. As meninas obtiveram melhor desempenho do que os meninos em todas as habilidades, tanto cognitivas quanto motoras, revelando desempenho muito melhor nos resultados de aprendizagem 
obtidos em relação às habilidades cognitivas. Duas formas diferentes de relacionar as habilidades cognitivas e motoras são feitas por cada um dos grupos de gênero e podem ser consideradas como estratégias diferentes por eles seguidas.

Neste caso, combinando relações e pontuações, as estratégias de aprendizagem das meninas são mostradas como mais eficazes. Esse achado por indicar o porquê mulheres tenham rendimento, neste estudo, não tenham sofrido desvantagens de seu rendimento apesar de enfrentarem diferenças amplas em termos de qualidade do ambiente de aprendizagem remota.

$\mathrm{Na}$ análise do efeito da covariável nível de curso na relação entre as variáveis independente e dependente, constatou-se maior rendimento médio dos estudantes de pós-graduação em relação aos da graduação. As pesquisas nesta área demonstram que mais da metade dos alunos que ingressa no nível superior revela dificuldades acadêmicas, havendo aumento de níveis de psicopatologias da população universitária (ALMEIDA; SOARES; FERREIRA, 1999; PARKER; COL, 2004; SANTOS, 2000, 2011).

Segundo Almeida (1998) e Santos (2004), as relações individuais e sociais e seu contexto exercidas durante o Ensino Básico, servem de referência para uma melhor compreensão do ajustamento acadêmico e da realização acadêmica dos estudantes do Ensino Superior. Nesse sentido, a qualidade de transição do Ensino Médio para o Ensino Superior vai depender tanto do desenvolvimento psicossocial do aluno, como também da instituição e dos mecanismos de apoio colocado à disposição deles. Nesse sentido, os alunos da graduação estão mais vulneráveis ao rendimento negativo devido a esta transição que muitas das vezes não é da melhor qualidade, também o nível de comprometimento e responsabilidade é menor nesta fase.

Na graduação, conforme Santos et al. (2011) o estudante possui uma maior autonomia que nem sempre encontrada no Ensino Básico, ou seja, precisará de dedicação e responsabilidade para ter um bom desemprenho. Dessa forma, nem sempre o estudante está preparado para suas responsabilidades acadêmicas, negligenciando assim sua formação. Por outro lado, estudantes de pós-graduação já possuem um objetivo formado, desejando ingressar em seu curso de pós-graduação almejado. Nesse sentido, possuem maior dedicação às suas atividades, o que pode surtir efeito positivo em seus rendimentos.

\section{Conclusão}

$\mathrm{O}$ isolamento social imposto pelas restrições advindas das medidas de controle da Pandemia de COVID-19 determinou uma abruta transição do ensino presencial praticado no ambiente escolar para a modalidade de ensino remoto em ambiente doméstico. Com isso, a oferta das condições objetivas para manutenção da qualidade da ambiência dos locais virtuais e presenciais de ensino e aprendizagem passou a depender da capacidade de cada estudante individual e privadamente.

Para os estudantes de Manaus, ainda que apenas parcialmente, a qualidade da ambiência de aprendizagem remota influenciou positivamente a autoavaliação de rendimento durante a Pandemia. Cada unidade de melhora na ambiência correspondeu a um incremento equivalente a até $2 / 3$ de unidade no rendimento do estudante. No entanto, essa relação parece ser particularmente importante e significativa apenas para os estudantes do gênero masculino e para estudantes de graduação. Mulheres e estudantes de pós-graduação parecem ser capazes de melhor se adaptarem às condições adversas do ensino remoto, em condições de isolamento domiciliar, durante a crise de saúde pública imposta pela Pandemia de COVID-19.

Considerando gênero e nível de curso, a qualidade da ambiência explica $1 / 5$ da variação do rendimento na avaliação pelos estudantes. Isso indica que outras variáveis também interferem no rendimento e que não estão associadas com às condições objetivas e do ambiente de estudo-aprendizagem.

O rendimento escolar foi considerado satisfatório na avaliação dos estudantes entrevistados, exceto no quesito interatividade, podendo ser este um impacto negativo decorrente do emprego deficitário de ferramentas e estratégias de comunicação no design e na operação dos ambientes virtuais de ensino e aprendizagem que se agrava em razão das deficiências relatadas na qualidade da ambiência.

Dessa forma, este estudo confirma que os desafios provocados pela transição educacional abrupta têm implicações negativas no desempenho e motivação dos estudantes, em particular no rendimento acadêmico. No contexto universitário, essas dificuldades são de diversas naturezas, passando tanto pelas questões individuais dos estudantes como também pelas novas exigências acadêmicas e do novo ambiente de ensino e aprendizagem, com influências diretas e indiretas no desempenho e desenvolvimento psicossocial dos estudantes.

\section{Referências}

ALMEIDA, L. S. Adaptação, rendimento e desenvolvimento dos estudantes do ensino superior. Estudo junto dos alunos do $1^{\circ}$ ano da Universidade do Minho. Braga. Minho: Universidade do Minho, 1998.

ALMEIDA, L.S.; SOARES, A.P.C.; FERREIRA, J.A.G. Adaptação, rendimento e desenvolvimento dos estudantes no Ensino Superior: construção, validação do questionário acadêmico de vivências acadêmicas relatórios de investigação. Methodus, v.3, n.5, p.3-20, 1999.

ASANOV, I. et al. Remote-learning, time-use, and mental health of Ecuadorian high-school students during the COVID-19 quarantine. World Develop., v.138, 2021. doi: 10.1016/j. worlddev.2020.105225

ATLAS ODS AMAZONAS. Proficiência dos estudantes $9^{\circ}$ ano do Ensino Fundamental dos municípios na Prova Brasil. Boletim do Atlas ODS Amazonas, v. 1, n. 5, p.1-2, 2019.

BLAZQUEZ-MERINO, M. et al. Gender comparative research on learning strategies applying the cognitive-motor model 
methodology and VISIR remote lab. Comput Appl Eng Educ., v. 27 , n.2019, p.869-884, 2019. doi: https://doi.org/10.1002/ cae. 22121

BRASIL. Ministério da Saúde. Secretaria de Atenção à Saúde. Núcleo Técnico da Política Nacional de Humanização. Brasília: MS, 2010.

CAVALCANTI, J.R. et al. COVID-19 no Brasil: Evolução da epidemia até a semana epidemiológica 20 de 2020. Epidemiol Serv. Saúde, v.29, n.4, 2020. doi: http://dx.doi.org/10.5123/ s1679-49742020000400010

CZERNIEWICZ, L. Inequality as higher education goes online. Networked learning. Springer, p. 95-106, 2018.

DIMAGGIO, P. et al. Digital inequality: From unequal access to differentiated use. In: SOCIAL INEQUALITY. Russell Sage Foundation, p.355-400, 2004.

ELALI, G.A. O ambiente da escola - o ambiente na escola: uma discussão sobre a relação escola-natureza em educação infantil. Estud. Psicol., v.8, n.2, p.309-319, 2003. doi: https://doi. org/10.1590/S1413-294X2003000200013

GOMES, M.P.; DUARTE, A.J. Desigualdade social e o direito à educação no brasil: reflexões a partir da reforma do ensino médio. Rev. Inter Ação, v.44, n.1, p.16-31, 2019. doi: https://doi. org/10.5216/ia.v44i1.55708

GUSSO, H.L. et al. Ensino superior em tempos de pandemia: diretrizes à gestão universitária. Educ. Soc., v.41, p.1-26, 2020. doi: https://doi.org/10.1590/ES.238957

LIMA. N.T.; BUSS, P.M; PAES-SOUSA, R.P. A pandemia de COVID-19: uma crise sanitária e humanitária. Cad. Saúde Pública, v. 36, n. 7, 2020. doi: https://doi.org/10.1590/0102311X00177020

MARTINS, V.; ALMEIDA, J. Educação em tempos de pandemia no Brasil: saberesfazeres escolares em exposição nas redes. Rev. Doc. Cibercultura, v.4, n.2, p.215-224, 2020. doi: https://doi. org/10.12957/redoc.2020.51026

PARKER, J.D.A. et al. Emotional intelligence and academic success examining the transition from high school to university. Personality and Individual Differences, v.36, p.163-172, 2004. doi: https://doi.org/10.1016/j.paid.2006.04.022

RONDINI. C.A; PEDRO, K.M; DUARTE, C.S. Pandemia da Covid-19 e o ensino remoto emergencial: Mudanças na prática pedagógica. Interfaces Cient., v.10, n.1, p.41-57, 2020. doi: https://doi.org/10.17564/2316-3828.2020v10n1p41-57

SANTOS, L.T.M. Vivências acadêmicas e rendimento escolar: estudo com alunos universitários do $1^{\circ}$ ano. Braga: Universidade do Minho, 2000.

SANTOS, A.A.A. et al. A relação entre vida acadêmica e a motivação para aprender em universitários. Psicol. Esc. Educ., v.15, n.2, p.283-290, 2011. doi: https://doi.org/10.1590/S141385572011000200010

SENKEVICS, A.S.; CARVALHO, M.P. O que você quer ser quando crescer?. Escolarização e gênero entre crianças de camadas populares urbanas. Rev. Bras. Estud. Pedagog., v.97, n.245, p.179-194, 2016. doi: https://doi.org/10.1590/S2176$6681 / 380613879$

SILVA, M. Indicadores de interatividade para o professor presencial e on-line. Rev. Diálogo Educ., v.4, n.12, p.93-109, 2004. doi: http://dx.doi.org/10.7213/rde.v4i12.6956

UNESCO. A Comissão Futuros da Educação da Unesco apela ao planejamento antecipado contra o aumento das desigualdades após a COVID-19. Paris: Unesco, 2020.

VOYER, D.; VOYER, S.D. Gender differences in scholastic achievement: a meta-analysis. Psychol. Bull., v.140, n. 4, p.11741204, 2014. doi: http://dx.doi.org/10.1037/a0036620 